(C)2009 IEEE. Personal use of this material is permitted. However, permission to reprint/republish this material for advertising or promotional purposes or for creating new collective works for resale or redistribution to servers or lists, or to reuse any copyrighted component of this work in other works must be obtained from the IEEE. 


\title{
Practical Stability and Controllability for Nonlinear Discrete Time-delay Systems
}

\author{
Zhan Su, Qingling Zhang and Wanquan Liu
}

\begin{abstract}
In this paper we study the practical asymptotic stability for a class of discrete-time time-delay systems via Razumikhin-type Theorems. Further estimations of the solution boundary and arrival time of the solution are also investigated based on practical stability. In addition, the proposed theorems are used to analyze the practical controllability of a general class of nonlinear discrete systems with input time delay. Some easy testing criteria for the uniform practical asymptotical stability are derived via Lyapunov function and Razumikhin technique. Finally a numerical example is given to illustrate the effectiveness of the proposed results.
\end{abstract}

\section{INTRODUCTION}

Since Lasalle first introduced the concept of practical stability in [1], it attracts much attention in control community. Many works on practical stability have been published with broad applications in different areas. Being much different from stability in terms of Lyapunov functions, practical stability, which stabilizes a system into a region of phase space, is a significant performance specification from engineering point of view, and are satisfactory in many applications for quality analysis. In practice, a system is actually unstable, just because the stable domain or the domain of the desired attractor is not large enough; or sometimes, the desired state of a system may be mathematically unstable, yet the system may oscillate sufficiently near to a state, in which the performance is still acceptable, i.e., it is stable in practice. For example, in practical communication or digital control systems, the signals of controller states, measurement outputs, and control inputs are quantized and then coded for transmission. A feedback law, which global asymptotically stabilizes a given system without quantization, will in general fail to guarantee global asymptotic stability of the closed-loop system, which arises in the presence of a quantizer with a finite number of values. Instead of global asymptotic stability, the practice stability can be obtained, where there is a region of attraction in the state and the steady state converges to a small limit cycle [2], [3], [4], [5], [6].

This work was supported by a grant from National Natural Science Foundation of China with grant number (60574011)

Z.Su is with Control Theorem and Engineering, College of Information Science and Engineering, Northeastern University, Shenyang, Liaoning province, 110004, PR China suzhan@foxmail. com

Q.Zhang is with the Institute of Systems Science, Northeastern University, Shenyang, Liaoning province, 110004, PR China qlzhang@mail. neu. edu. cn

W.Liu is with the Department of Computing, Curtin University of Technology, Perth WA 6102, Australia W. Liu@curtin.edu. cn
On the other hand, it is well known that for more than one hundred years Lyapunov's direct method has been the primary technique for dealing with stability problems in difference equations. However, the construction of Lyapunov's function is much more difficult for time-delay systems than for non-delay systems. Such difficulties can be overcome via using Lyapunov functions and Razumikhin techniques. It should be pointed out that the Razumikhintype method could deal with the time-delay problem effectively and are easier to apply in general, therefore such a method has been a main technique for analyzing the stability for time-delay systems [7], [8], [9], [10]. To the best of our knowledge, the studies of Razumikhin-type method on practical stability for discrete time-delay systems has not been investigated. Motivated by results in [9], we will study the Razumikhin-type theorem on practical asymptotic stability for a class of discrete time-delay system in this paper. Also estimations of the solution boundary and arrival time of the solution are discussed. Consequently, the proposed theorems are used to study the practical controllability of a general class of nonlinear discrete systems with input time delay. Some easy-testing criteria for the uniform practical asymptotical stability are obtained via Lyapunov function and Razumikhin technique.

This paper is organized as follows. In Section II, some definitions and preliminaries are introduced. In Section III, some criteria for uniform practical asymptotical stability of discrete-time systems with finite delay are derived via Lyapunov functions and Razumikhin-technique. In Section IV, estimation of the solution boundary and arrival time of the solution are investigated in terms of practical stability. In Section V, the proposed theorems are used to analyze the practical controllability for a general class of nonlinear discrete systems with input time delay. In Section VI, a numerical example is given to illustrate the effectiveness of main results obtained from the Section V. The last section gives some conclusions.

Notation: $\mathbb{R}^{N}$ denotes the $N$-dimensional Euclidean space, $\mathbb{Z}^{+}$is the set of nonnegative integer. Let $I_{d}=\{-d,-d+$ $1, \ldots,-1,0\}$ with some integer $d \geq 0, I_{d}^{1}=I_{d} \cup\{1\}$, $\Upsilon\left(I_{d}, \mathbb{R}^{N}\right)=\left\{\varphi_{d}=\left(\varphi^{T}(0), \varphi^{T}(-1), \ldots, \varphi^{T}(-d)\right)^{T} \mid \varphi: I_{d} \rightarrow\right.$ $\left.\mathbb{R}^{N}\right\}$. For all $\varphi_{d} \in \Upsilon\left(I_{d}, \mathbb{R}^{N}\right)$, define the norm of $\varphi_{d}$ as $\left\|\varphi_{d}\right\|=\max _{s \in I_{d}}|\varphi(s)|$, where $|\cdot|$ stands for any norm in $\mathbb{R}^{N}$. Let $\Upsilon_{B}\left(I_{d}, \mathbb{R}^{N}\right)=\Upsilon\left(I_{d}, \mathbb{R}^{N}\right) \cap\left\{\varphi_{d}: \varphi(s) \in B, s \in I_{d}\right\}$, where $B$ is an open ball. 


\section{PRELIMINARIES}

Consider a general class of nonlinear discrete-time systems with finite delay as follows:

$$
X(k+1)=F\left(k, X_{d}(k)\right), \quad k \in \mathbb{Z}^{+},
$$

where $d \geq 0$ is an integer, $X(k) \in \mathbb{R}^{N}, \quad X_{d}(k)=$ $\left(X^{T}(k), X^{T}(k-1), \ldots, X^{T}(k-d)\right)^{T}, F: \mathbb{Z}^{+} \times \Upsilon_{B}\left(I_{d}, \mathbb{R}^{N}\right) \rightarrow$ $\mathbb{R}^{N}$. We assume that $F$ satisfies certain conditions to guarantee the global existence and uniqueness of solutions, and $F(k, 0)=0$ for $k \in \mathbb{Z}^{+}$. Thus system (1) has zero solution $X(\cdot) \equiv 0$. For any $k_{0} \in \mathbb{Z}^{+}$and any given initial function $X_{0} \in \Upsilon_{B}\left(I_{d}, \mathbb{R}^{N}\right)$, the solution of the systems (1) denoted by $X\left(k ; k_{0}, X_{0}\right)$ satisfies (1) for all integers $k \geq k_{0}$, and $X\left(k_{0}+s ; k_{0}, X_{0}\right)=X_{0}(s)$ for all $s \in I_{d}$. We further assume that there exists a constant $L>0$ such that for all $\varphi_{d} \in \Upsilon_{B}\left(I_{d}, \mathbb{R}^{N}\right)$,

$$
\left|F\left(k, \varphi_{d}\right)\right| \leq L\left\|\varphi_{d}\right\|, \quad \forall k \in \mathbb{Z}^{+} .
$$

We introduce the following definitions.

Definition 2.1: [9] A wedge function is a continuous strictly increasing function $W: \mathbb{R}^{+} \rightarrow \mathbb{R}^{+}$with $W(0)=0$.

Definition 2.2: System (1) is called to be

$\left(\mathrm{PS}_{1}\right)$ practically stable (P.S.) if for given $(\alpha, \beta)$ with $0<$ $\alpha<\beta$ and some $k_{0} \in \mathbb{Z}^{+}$, we have $\left\|X_{0}\right\|<\alpha$ implies $\left|X\left(k ; k_{0}, X_{0}\right)\right|<\beta, k \geq k_{0}$;

$\left(\mathrm{PS}_{2}\right)$ uniformly practically stable (U.P.S.) if P.S. holds for all $k_{0} \in \mathbb{Z}^{+}$

$\left(\mathrm{PS}_{3}\right)$ practically asymptotic stable (P.A.S.) if P.S. holds, and for each $\varepsilon \in(0, \beta)$, there exists a positive number $K=$ $K\left(k_{0}, \alpha, \varepsilon\right)$ such that $\left\|X_{0}\right\|<\alpha$ implies $\left|X\left(k ; k_{0}, X_{0}\right)\right|<$ $\varepsilon, k \geq k_{0}+K$

$\left(\mathrm{PS}_{4}\right)$ uniformly practically asymptotic stable (U.P.A.S.) if P.A.S. holds for all $k_{0} \in \mathbb{Z}^{+}$.

Definition 2.3: For a function $V: \mathbb{Z}^{+} \times \mathbb{R}^{N} \rightarrow \mathbb{R}^{+}$, define:

$$
\begin{aligned}
\Delta V(k, X(k)) & \triangleq V(k+1, X(k+1))-V(k, X(k)) \\
& =V\left(k+1, F\left(k, X_{d}(k)\right)\right)-V(k, X(k)) .
\end{aligned}
$$

\section{RAZUMIKHIN-TYPE THEOREMS}

In this section we will prove the Razumikhin-type theorems with an aim of analyzing the uniformly practical asymptotic stability (U.P.A.S.) for a general class of nonlinear discrete-time systems with finite delay. We present the following result first for system (1). For the sake of brevity, we denote $X=X(k)$ and $X_{d}=X_{d}(k)$.

Theorem 3.1: Given positive scalars $\alpha$ and $\beta$. Assume that scalars $\pi_{1}, \pi_{2}, \pi_{3}$ with $0<\pi_{1} \leq \pi_{2}, \pi_{3}>0$ are all arbitrary. If there exist a scalar $\gamma>0$, a Lyapunov function $V: \mathbb{Z}^{+} \times \mathbb{R}^{N} \rightarrow \mathbb{R}^{+}$, and wedge functions $W_{i}(\cdot)(i=1,2,3)$, such that

(i) $\quad W_{1}(|X|) \leq V(k, X) \leq W_{2}(|X|)$;

(ii) $\Delta V(k, X) \leq-W_{3}(|X(k+1)|)+\pi_{3}$ for $\varepsilon_{0} \leq\left\|X_{d}\right\| \leq \rho_{0}$,

provided $\varepsilon_{0} \leq \rho_{0}, V(k+s, X(k+s)) \leq \min \left\{\pi_{2}, V(k+1, X(k+\right.$ 1) $)+\gamma\}$ for $s \in I_{d}^{1}$, and $\pi_{1} \leq V(k+1, X(k+1))$. Where $\varepsilon_{0}=$
$L^{-1} \alpha, \rho_{0}=\max \left\{\beta, W_{1}^{-1}\left(W_{2}(\alpha)\right)\right\}, L$ is defined by (2). Then, for the balls:

$B_{1}=\left\{X: V(k, X)<W_{1}(\beta)\right\} ; \quad B_{2}=\left\{X: V(k, X)<W_{2}(\alpha)\right\}$.

We have (1) $B_{2}$ is an invariable set; (2) If $W_{2}(\alpha)<W_{1}(\beta)$, then $B_{1}$ is an invariable set and there exists a positive number $K=K(\alpha, \beta)$ such that for any $k_{0} \in \mathbb{Z}, X_{0} \in \Upsilon_{B_{1}}\left(I_{d}, \mathbb{R}^{N}\right)$ implies $\forall k \geq k_{0}+K, X\left(k ; k_{0}, X_{0}\right) \in B_{2}$.

Proof. (1) For each $X_{0} \in \Upsilon_{B_{2}}\left(I_{d}, \mathbb{R}^{N}\right)$, we have $X\left(k ; k_{0}, X_{0}\right) \in$ $B_{2}$ for $k_{0}-d \leq k \leq k_{0}$. We claim that for all $k \geq k_{0}, X=$ $X\left(k ; k_{0}, X_{0}\right) \in B_{2}$.

Suppose this is not true. Then there exist some $k^{1} \geq k_{0}$ such that $X \in B_{2}$ for all $k_{0}-d \leq k \leq k^{1}$, and

$$
V\left(k^{1}+1, X\left(k^{1}+1\right)\right) \geq W_{2}(\alpha),
$$

and consequently,

$\Delta V\left(k^{1}, X\left(k^{1}\right)\right)=V\left(k^{1}+1, X\left(k^{1}+1\right)\right)-V\left(k^{1}, X\left(k^{1}\right)\right)>0$.

On the other hand, by condition (i), we have $W_{1}(|X|)<$ $W_{2}(\alpha)$ for $k_{0}-d \leq k \leq k^{1}$, which implies $\left\|X_{d}\right\| \leq \rho_{0}$ for $k_{0} \leq k \leq k^{1}$. It follows from (2), (3) and condition (i) that $\alpha \leq\left|X\left(k^{1}+1\right)\right| \leq L\left\|X_{d}\left(k^{1}\right)\right\| \leq L \rho_{0}$, which implies $\varepsilon_{0} \leq$ $\left\|X_{d}\left(k^{1}\right)\right\| \leq \rho_{0}, \varepsilon_{0} \leq \rho_{0}$. Let $0<\pi_{1} \leq W_{2}(\alpha) \leq W_{2}\left(L \rho_{0}\right) \leq \pi_{2}$, and $0<\pi_{3}<W_{3}(\alpha)$. Then, it follows from (3) that $\pi_{1} \leq$ $V\left(k^{1}+1, X\left(k^{1}+1\right)\right)$, and for $\gamma>0, \forall s \in I_{d}^{1}$, there holds

$$
\begin{aligned}
& \left\{\begin{array}{l}
V\left(k^{1}+s, X\left(k^{1}+s\right)\right)<\pi_{2} \\
V\left(k^{1}+s, X\left(k^{1}+s\right)\right)<V\left(k^{1}+1, X\left(k^{1}+1\right)\right)+\gamma
\end{array}\right. \\
& V\left(k^{1}+s, X\left(k^{1}+s\right)\right) \\
& \leq \min \left\{\pi_{2}, V\left(k^{1}+1, X\left(k^{1}+1\right)\right)+\gamma\right\} .
\end{aligned}
$$

By condition (ii), we have

$$
\Delta V\left(k^{1}, X\left(k^{1}\right)\right) \leq-W_{3}\left(\left|X\left(k^{1}+1\right)\right|\right)+\pi_{3}<0 .
$$

This is a contradiction. Thus for all $k \geq k_{0}, X \in B_{2}$, i.e., $B_{2}$ is an invariable set.

(2) If $W_{2}(\alpha)<W_{1}(\beta)$, we first prove that $B_{1}$ is an invariable set. In fact, $\rho_{0}=\beta$, and $\varepsilon_{0}=L^{-1} \alpha<L^{-1} W_{2}^{-1}\left(W_{1}(\beta)\right)$. Similar to the proof of (1), one can derive that, $X_{0} \in \Upsilon_{B_{1}}\left(I_{d}, \mathbb{R}^{N}\right)$ implies $X \in B_{1}$ for all $k \geq k_{0}$.

Next, we will find an integer $K=K(\alpha, \beta)>0$ such that for all $k_{0} \in \mathbb{Z}^{+}, X_{0} \in \Upsilon_{B_{1}}\left(I_{d}, \mathbb{R}^{N}\right)$ implies $X\left(k ; k_{0}, X_{0}\right) \in B_{2}$ for all $k \geq k_{0}+K$.

Assume that $0<\pi_{1} \leq W_{2}(\alpha)<W_{1}(\beta) \leq \pi_{2}, 0<\pi_{3}<$ $(1 / 2) W_{3}(\alpha)$. Let $\hat{N}$ be the first positive integer such that

$$
W_{1}(\beta)<W_{2}(\alpha)+\hat{N} \gamma
$$

For each $i \in\{0,1, \ldots, \hat{N}\}$, let

$$
k_{i}=k_{0}+i\left(d+\left[\frac{W_{1}(\beta)}{\pi_{3}}\right]\right),
$$

where $[\cdot]$ denotes the greatest integer function, $\gamma$ is depended on $\pi_{1}$ and $\pi_{3}$. We show that for all $i \in\{0,1, \ldots, \hat{N}\}$,

$$
V(k, X)<W_{2}(\alpha)+(\hat{N}-i) \gamma, \quad \forall k \geq k_{i} .
$$


Obviously, it follows (4) that (5) holds for $i=0$ since $X \in B_{1}$ for all $k \geq k_{0}$. Suppose (5) holds for some $i \in\{0,1, \ldots, \hat{N}-$ $1\}$, we aim to show that (5) also holds for $i+1$, i.e.,

$$
V(k, X)<W_{2}(\alpha)+(\hat{N}-i-1) \gamma, \quad \forall k \geq k_{i+1} .
$$

We decompose our proof into 2 steps.

Step 1 . We show that there does exist some $k^{\prime} \in\left[k_{i}+d, k_{i+1}\right]$ such that

$$
V\left(k^{\prime}, X\left(k^{\prime}\right)\right)<W_{2}(\alpha)+(\hat{N}-i-1) \gamma
$$

Suppose this is not true, for all $k \in\left[k_{i}+d, k_{i+1}\right]$, we would have

$$
V(k, X) \geq W_{2}(\alpha)+(\hat{N}-i-1) \gamma .
$$

Noting the assumption that (5) holds for some $i \in$ $\{0,1, \ldots, \hat{N}-1\}$, then, for all $k \in\left[k_{i}+d, k_{i+1}-1\right], s \in I_{d}^{1}$, from (7) we have

$$
\begin{aligned}
V(k+s, X(k+s)) & <W_{2}(\alpha)+(\hat{N}-i) \gamma \\
& \leq V(k+1, X(k+1))+\gamma .
\end{aligned}
$$

On the other hand, for all $k \in\left[k_{i}+d, k_{i+1}-1\right]$, it follows from condition (i), (2) and (7) that $W_{2}(\alpha) \leq V(k+1, X(k+1)) \leq$ $W_{2}(|X(k+1)|)$, which implies that $\alpha \leq|X(k+1)| \leq L \rho_{0}$, $\varepsilon_{0} \leq\left\|X_{d}(k)\right\| \leq \rho_{0}, \varepsilon_{0} \leq \rho_{0}$. Then, for all $k \in\left[k_{i}+d, k_{i+1}-1\right]$, $V(k+s, X(k+s)) \leq \pi_{2}, s \in I_{d}^{1}$, and it follows from (7) that $V(k+1, X(k+1)) \geq \pi_{1}$. By condition (ii), for all $k \in\left[k_{i}+\right.$ $\left.d, k_{i+1}-1\right]$,

$$
\Delta V(k, X) \leq-W_{3}(|X(k+1)|)+\pi_{3}<-\pi_{3} .
$$

Hence, we have

$$
\begin{aligned}
V\left(k_{i+1}, X\right) & \leq V\left(k_{i}+d, X\left(k_{i}+d\right)\right)-\pi_{3}\left(k_{i+1}-k_{i}-d\right) \\
& <W_{1}(\beta)-\pi_{3}\left[\frac{W_{1}(\beta)}{\pi_{3}}\right]<0 .
\end{aligned}
$$

This is a contradiction to the definition of Lyapunov function $V$. Thus, there does exist some $k^{\prime} \in\left[k_{i}+d, k_{i+1}\right]$ such that (6) holds.

Step 2. We show that

$$
V(k, X)<W_{2}(\alpha)+(\hat{N}-i-1) \gamma, \quad \forall k \geq k^{\prime} .
$$

In fact, suppose this is not true, there must be some $k_{1}^{\prime} \geq k^{\prime}$ such that

$$
\begin{array}{r}
V\left(k_{1}^{\prime}, X\left(k_{1}^{\prime}\right)\right)<W_{2}(\alpha)+(\hat{N}-i-1) \gamma, \quad \text { and } \\
V\left(k_{1}^{\prime}+1, X\left(k_{1}^{\prime}+1\right)\right) \geq W_{2}(\alpha)+(\hat{N}-i-1) \gamma,
\end{array}
$$

and hence we have $\Delta V\left(k_{1}^{\prime}, X\left(k_{1}^{\prime}\right)\right)>0$. On the other hand, $\pi_{1} \leq W_{2}(\alpha) \leq V\left(k_{1}^{\prime}+1, X\left(k_{1}^{\prime}+1\right)\right), V\left(k_{1}^{\prime}+s, X\left(k_{1}^{\prime}+s\right)\right) \leq$ $\pi_{2}$. Noting the assumption that (5) holds for some $i \in$ $\{0,1, \ldots, \hat{N}-1\}$, then, for $s \in I_{d}^{1}$, we have

$$
\begin{aligned}
V\left(k_{1}^{\prime}+s, X\left(k_{1}^{\prime}+s\right)\right) & <W_{2}(\alpha)+(\hat{N}-i) \gamma \\
& \leq V\left(k_{1}^{\prime}+1, X\left(k_{1}^{\prime}+1\right)\right)+\gamma
\end{aligned}
$$

From condition (i), (2) and (9), we have $W_{2}(\alpha) \leq V\left(k_{1}^{\prime}+\right.$ $\left.1, X\left(k_{1}^{\prime}+1\right)\right) \leq W_{2}\left(\left|X\left(k_{1}^{\prime}+1\right)\right|\right)$, and hence, $\alpha \leq\left|X\left(k_{1}^{\prime}+1\right)\right| \leq$
$L \rho_{0}, \varepsilon_{0} \leq\left\|X_{d}\left(k_{1}^{\prime}\right)\right\| \leq \rho_{0}, \varepsilon_{0} \leq \rho_{0}$. With condition (ii), one can derive that

$$
\Delta V\left(k_{1}^{\prime}, X\left(k_{1}^{\prime}\right)\right) \leq-W_{3}\left(\left|X\left(k_{1}^{\prime}+1\right)\right|\right)+\pi_{3} \leq-\pi_{3}<0 .
$$

This is a contradiction again to the definition of Lyapunov function $V$. Thus (8) holds, and consequently, (5) holds for all $i \in\{0,1, \ldots, \hat{N}\}$. Therefore, we obtain that $X \in B_{2}$ for all $k \geq k_{\hat{N}}=k_{0}+K$, where $K=\hat{N}\left(d+\left[\frac{W_{1}(\beta)}{\pi_{3}}\right]\right)$ is independent of $k_{0}$ and $X_{0}$.

From Theorem 3.1, we have following corollary.

Corollary 3.2: Given positive scalars $\alpha$ and $\beta$. Assume that $\hat{P}(s) \in C\left(\mathbb{R}^{+}, \mathbb{R}^{+}\right)$with $\hat{P}(s)>s$ for $s>0$. If there exist a Lyapunov function $V: \mathbb{Z}^{+} \times \mathbb{R}^{N} \rightarrow \mathbb{R}^{+}$, and wedge functions $W_{i}(\cdot)(i=1,2,3)$, satisfying the conditions (i) in Theorem 3.1 and the following condition (ii)'

$$
\text { (ii) }^{\prime} \quad \Delta V(k, X) \leq-W_{3}(|X(k+1)|) \text { for } \varepsilon_{0} \leq\left\|X_{d}\right\| \leq \rho_{0},
$$

provided $\varepsilon_{0} \leq \rho_{0}, V(k+s, X(k+s))<\hat{P}(V(k+1, X(k+1)))$ for $s \in I_{d}^{1}$. Where $\varepsilon_{0}=L^{-1} \alpha, \rho_{0}=\max \left\{\beta, W_{1}^{-1}\left(W_{2}(\alpha)\right)\right\}, L$ is defined by (2). Then, the conclusion of Theorem 3.1 still holds.

Proof. For any $0<\pi_{1} \leq \pi_{2}$, and any $\pi_{3}>0$, choose $\gamma \in$ $\left(0, \inf \left\{\hat{P}(s)-s: \pi_{1} \leq s \leq \pi_{2}\right\}\right)$. Then, if $V(k+s, X(k+s)) \leq$ $\min \left\{\pi_{2}, V(k+1, X(k+1)+\gamma)\right\}$ for $s \in I_{d}^{1}$, and $\pi_{1} \leq V(k+$ $1, X(k+1))$, we have

$$
\begin{aligned}
V(k+s, X(k+s)) & \leq V(k+1, X(k+1))+\gamma \\
& <\hat{P}(V(k+1, X(k+1))),
\end{aligned}
$$

for $s \in I_{d}^{1}$. Hence, by condition (ii) ${ }^{\prime}$, we have

$$
\Delta V(k, X) \leq-W_{3}(|X(k+1)|) \leq-W_{3}(|X(k+1)|)+\pi_{3} .
$$

Then, the condition (i) and (ii) in Theorem 3.1 are both satisfied. Therefore, the result follows.

By employing Theorem 3.1 and Corollary 3.2, we obtain the following Razumikhin-type theorems for the U.P.A.S. of the zero solution of systems (1).

Theorem 3.3: For given scalar pair $(\alpha, \beta)$ with $0<\alpha<\beta$, $\varepsilon \in(0, \beta)$ is arbitrary. Assume that scalars $\pi_{1}, \pi_{2}, \pi_{3}$ with $0<\pi_{1} \leq \pi_{2}, \pi_{3}>0$ are all arbitrary, $\hat{P}(s) \in C\left(\mathbb{R}^{+}, \mathbb{R}^{+}\right)$with $\hat{P}(s)>s$ for $s>0$. If there exist a Lyapunov function $V$ : $\mathbb{Z}^{+} \times \mathbb{R}^{N} \rightarrow \mathbb{R}^{+}$, wedge functions $W_{i}(\cdot)(i=1,2,3)$, satisfying

$$
\begin{array}{ll}
\text { (i) } & W_{2}(\alpha) \leq W_{1}(\beta) ; \\
\text { (ii) } & W_{1}(|X|) \leq V(k, X) \leq W_{2}(|X|) ;
\end{array}
$$

and either the following conditions (iii) $)_{a}$ or(iii) $)_{b}$ for $\varepsilon_{0} \leq$ $\left\|X_{d}\right\| \leq \rho_{0}, \varepsilon_{0} \leq \rho_{0}$ :

$$
\begin{aligned}
& \Delta V(k, X) \leq-W_{3}(|X(k+1)|)+\pi_{3}, \text { provided } \\
& V(k+s, X(k+s)) \leq \min \left\{\pi_{2}, V(k+1, X(k+1))+\gamma\right\} \\
& \text { for } s \in I_{d}^{1}, \text { and } \pi_{1} \leq V(k+1, X(k+1)) ; \\
& \Delta V(k, X) \leq-W_{3}(|X(k+1)|), \text { provided for } s \in I_{d}^{1}, \\
& V(k+s, X(k+s))<\hat{P}(V(k+1, X(k+1))) .
\end{aligned}
$$

Where $\varepsilon_{0}=L^{-1} W_{2}^{-1}\left(W_{1}(\varepsilon)\right), \rho_{0}=\beta, L$ is defined by (2). Then the zero solution of systems (1) is U.P.A.S.. 


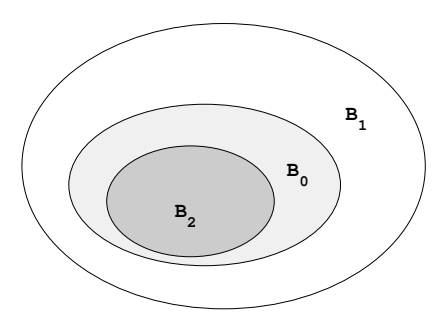

Fig. 1. The relationship of the balls $B_{0}, B_{1}$ and $B_{2}$

Proof. Define the following balls:

$$
\begin{aligned}
& B_{0}=\left\{X: V(k, X)<W_{2}(\alpha)\right\} ; \\
& B_{1}=\left\{X: V(k, X)<W_{1}(\beta)\right\} ; \\
& B_{2}=\left\{X: V(k, X)<W_{1}(\varepsilon)\right\} .
\end{aligned}
$$

By condition (i), $B_{0} \subseteq B_{1}$, as shown in Fig 1 . Since $\varepsilon_{0}=L^{-1} W_{2}^{-1}\left(W_{1}(\varepsilon)\right)<L^{-1} W_{2}^{-1}\left(W_{1}(\beta)\right)$, then, by Theorem 3.1 and Corollary 3.2, we can assert that, both $B_{1}$ and $B_{2}$ are invariant sets, and there exists a positive number $K=K(\alpha, \varepsilon)$ such that for any $k_{0} \in \mathbb{Z}$, $X_{0} \in \Upsilon_{B_{0}}\left(I_{d}, \mathbb{R}^{N}\right)$ implies $\forall k \geq k_{0}+K, X\left(k ; k_{0}, X_{0}\right) \in B_{2}$. By condition (ii), $|X|<\alpha$ implies $X \in B_{0} ; X \in B_{1}$ implies $|X|<\beta ; X \in B_{2}$ implies $|X|<\varepsilon$. Then, for any $k_{0} \in \mathbb{Z}$, ||$X_{0} \|<\alpha$ implies $\forall k \geq k_{0}+K,\left|X\left(k ; k_{0}, X_{0}\right)\right|<\varepsilon$, i.e., the zero solution of the systems (1) is U.P.A.S..

\section{ESTIMATION OF SOLUTION BOUNDARY AND ARRIVAL TIME}

In the previous section, without condition (i), for the balls $B_{0}=\left\{X: V(k, X)<W_{2}(\alpha)\right\}$ and $B_{1}=\left\{X: V(k, X)<W_{1}(\beta)\right\}$, let $\varepsilon_{0}=L^{-1} W_{2}^{-1}\left(W_{1}(\beta)\right), \rho_{0}=\max \left\{\beta, W_{1}^{-1}\left(W_{2}(\alpha)\right)\right\}$. From conditions (ii), (iii) $)_{a} \sim(\text { iii) })_{b}$, we can obtain the conclusion that $B_{1}$ is an invariant set and the trajectory of the solution of system (1), which starts from $B_{0}$, will fall into $B_{1}$ in finite time. In addition, with the assumption of condition (i), all trajectories of the considered solutions, which exit from the ball $B_{0}$, will take the ball $B_{1}$ to be their boundary. Thus, in the proposed theorems, as long as $\varepsilon_{0} \leq L^{-1} W_{2}^{-1}\left(W_{1}(\beta)\right)$, and $\Delta V(k, X) \leq 0$ in conditions (iii) $)_{a} \sim($ iii) $b$, the system is U.P.S.. Along the light of the above analysis, it is more conveniently to apply Theorem 3.1 and Corollary 3.2 in this paper to estimate relations between balls $B_{1}$ and $B_{2}$ by utility of information on $\varepsilon_{0} \leq\left\|X_{d}(k)\right\| \leq \rho_{0}$, which are not mentioned in Theorem 1, Corollary 1 and Corollary 2 in [9]. We give the following theorem to estimate both the boundary of the solution of system (1) and arrival time $K$, after which the solution exists from the given ball $\{X:\|X\|<\alpha\}$ falls into the region $\{X:\|X\|<\varepsilon\}$, where $0<\varepsilon<\alpha$.

Theorem 4.1: Given scalars $\alpha, \varepsilon$ with $0<\varepsilon<\alpha, \sigma_{1}>1$. If there exist a Lyapunov function $V: \mathbb{Z}^{+} \times \mathbb{R}^{N} \rightarrow \mathbb{R}^{+}$, wedge functions $W_{i}(\cdot)(i=1,2,3)$, satisfying

$$
\begin{aligned}
& W_{1}(|X|) \leq V(k, X) \leq W_{2}(|X|) \\
& \Delta V(k, X) \leq-W_{3}(|X(k+1)|) \text { for }\left\|X_{d}\right\| \leq \rho_{0}, \text { provided } \\
& V(k+s, X(k+s))<\sigma_{1}(V(k+1, X(k+1))) \text { for } s \in I_{d}^{1} .
\end{aligned}
$$

Then

(1) $\hat{\beta}=W_{1}^{-1}\left(W_{2}(\alpha)\right)$;

(2) $K=k_{0}+\bar{N}_{1}\left(d+\bar{N}_{2}\right)$.

Where

$\bar{N}_{1}=\left\{\begin{array}{lc}\frac{W_{2}(\alpha)+\left(\sigma_{1}-2\right) W_{1}(\varepsilon)}{\left(\sigma_{1}-1\right) W_{1}(\varepsilon)}, & \frac{W_{2}(\alpha)-W_{1}(\varepsilon)}{\left(\sigma_{1}-1\right) W_{1}(\varepsilon)} \text { is integer; } \\ {\left[\frac{W_{2}(\alpha)-W_{1}(\varepsilon)}{\left(\sigma_{1}-1\right) W_{1}(\varepsilon)}\right],} & \text { otherwise, }\end{array}\right.$
$\bar{N}_{2}=\left\{\begin{array}{lc}\frac{2 W_{2}(\alpha)}{W_{3}\left(W_{2}^{-1}\left(W_{1}(\varepsilon)\right)\right)}+1, & \frac{2 W_{2}(\alpha)}{W_{3}\left(W_{2}^{-1}\left(W_{1}(\varepsilon)\right)\right)} \text { is integer } \\ {\left[\frac{2 W_{2}(\alpha)}{W_{3}\left(W_{2}^{-1}\left(W_{1}(\varepsilon)\right)\right.}\right],} & \text { otherwise }\end{array}\right.$

[.] denotes the greatest integer function, $\rho_{0}=W_{1}^{-1}\left(W_{2}(\alpha)\right)$, $\hat{\beta}$ is the estimation of the solution boundary of system (1), and $K$ is the time that the solution exists from the given ball $\{X:\|X\|<\alpha\}$ and falls into the region $\{X:\|X\|<\varepsilon\}$.

Proof. (1) In Theorem 3.1, we let $W_{1}(\hat{\beta})=W_{2}(\alpha)$. Then, $\varepsilon_{0}=$ $L^{-1} \alpha, \rho_{0}=\hat{\beta}$, and $B_{1}=B_{2}=\left\{X: V<W_{1}(\hat{\beta})\right\}$. It follows from Theorem 3.1 that the solution starts from $B_{2}$ can not exits from $B_{1}$, which implies that the solution starting from set $\{X:\|X\|<\alpha\}$ will have a boundary $\hat{\beta}=W_{1}^{-1}\left(W_{2}(\alpha)\right)$. (2) In Theorem 3.1, we let $B_{1}=\left\{X: V(X)<W_{2}(\alpha)\right\}$, and $B_{2}=\left\{X: V(X)<W_{1}(\varepsilon)\right\}$. Noting that $\varepsilon_{0}=L^{-1} W_{2}^{-1}\left(W_{1}(\varepsilon)\right)$ and $\rho_{0}=W_{1}^{-1}\left(W_{2}(\alpha)\right)$. Let $\hat{P}(s)=\sigma_{1} s$, then $\hat{P}(s)$ has the required property in Corollary 3.2. There exist scalars $\delta_{1}>0$ and $\delta_{2} \in(0,1 / 2)$, such that $\frac{W_{2}(\alpha)-W_{1}(\varepsilon)}{\left(\sigma_{1}-1\right) W_{1}(\varepsilon)}<\frac{W_{2}(\alpha)-W_{1}(\varepsilon)}{\left(\sigma_{1}-1\right) W_{1}(\varepsilon)-\delta_{1}}<$ $\bar{N}_{1}$, and $\frac{2 W_{2}(\alpha)}{W_{3}\left(W_{2}^{-1}\left(W_{1}(\varepsilon)\right)\right)}<\frac{W_{2}(\alpha)}{\delta_{2} W_{3}\left(W_{2}^{-1}\left(W_{1}(\varepsilon)\right)\right)}<\bar{N}_{2}$. Under the similar process of Theorem 3.1 and Corollary 3.2, for $\varepsilon \leq$ $\|X\|<\alpha$, let $\gamma=\left(\sigma_{1}-1\right) W_{1}(\varepsilon)-\delta_{1} \in(0, \inf (\hat{P}(V)-V))$ and $\pi_{3}=\delta_{2} W_{3}\left(W_{2}^{-1}\left(W_{1}(\varepsilon)\right)\right)$, one can derive the conclusion of (2).

\section{PRACTICAL CONTROLLABILITY}

In this section we will use the results in previous sections to study the practical controllability for a general class of nonlinear discrete systems with input time delay. Consider the following system:

$$
x(k+1)=f(k, x(k))+\sum_{i=0}^{d} B(k-i) u(k-i)
$$

where $f: \mathbb{Z}^{+} \times \mathbb{R}^{n} \rightarrow \mathbb{R}^{n}, B: \mathbb{Z}^{+} \times \mathbb{R}^{n} \rightarrow \mathbb{R}^{n \times m}, i=1, \ldots, d$, $u(k) \in \mathbb{R}^{m}$ is input, and is supposed to guarantee the existence and uniqueness of the solution. This type of model is generally studied in networked control systems (NCSs). We first introduce the following definitions:

Definition 5.1: System (10) is called to be

$\left(\mathrm{PC}_{1}\right)$ uniformly practically controllable (U.P.C.) with respect to $(\alpha, \beta), 0<\alpha<\beta$, if there exist finite time $K$ and a control $u(\cdot)$ defined on $\left[k_{0}, K\right]$ such that all the solutions $x(k)=x\left(k ; k_{0}, x_{0}, u\right)$ that exit from $\left\{x \in \mathbb{R}^{n}:\left\|x_{0}\right\|<\alpha\right\}$ 
will enter into a bounded region $\left\{x \in \mathbb{R}^{n}:\left\|x_{d}\right\|<\beta\right\}$ at time $\mathrm{K}$ instant for all $k_{0} \in \mathbb{Z}^{+}$;

$\left(\mathrm{PC}_{2}\right)$ uniformly practically asymptotic controllable (U.P.A.C.) with respect to $(\alpha, \beta), 0<\alpha<\beta$, if U.P.C. holds, and for each $\varepsilon \in(0, \beta)$, there exists a positive number $K=K\left(k_{0}, \alpha, \varepsilon\right)$ such that $\left\|x_{0}\right\|<\alpha$ implies $\left|x\left(k ; k_{0}, x_{0}, u\right)\right|<\varepsilon$ for all $k_{0} \in \mathbb{Z}^{+}$.

For system (10), we have the following theorem on U.P.A.C. with respect to $(\alpha, \beta)$.

Theorem 5.2: Assume that there exists a control law $u(k)$ such that system (10) can be expressed by the form of (1), and the conditions of Theorem 3.3 are satisfied. Then, system (10) is U.P.A.C.with respect to $(\alpha, \beta)$.

In system (10), let $\hat{f}(k, x(k))=f(k, x(k))+B(k) u(k)$. Suppose that $\|\hat{f}(k, x(k))\| \leq\left\|\Psi_{0}(k)\right\|\|x(k)\|$. Adopt the feedback control law $u(k)=F(k, x(k)) x(k)$, and let $\Psi_{i}(k)=B(k-$ i) $F(k-i, x(k-i))$, where $F(k, x(k))$ is the control gain matrix, $\Psi_{0}(k)$ and $\Psi_{i}(k)$ are of compatible dimensions. Consequently, the closed-loop system of (10) has the following form:

$$
x(k+1)=\hat{f}(k, x(k))+\sum_{i=1}^{d} \Psi_{i}(k) x(k-i),
$$

Let $\lambda_{\max }(\cdot)$ and $\lambda_{\min }(\cdot)$ be the maximum eigenvalue and the minimum eigenvalue of a real symmetric matrix, respectively. $\|\cdot\|_{2}$ stands for the Euclidean vector norm or the 2norm of a matrix. Then, we have the following corollary.

Corollary 5.3: If there exists $F(k)$ such that

$$
\sup _{k \in \mathbb{Z}} \sum_{i=0}^{d}\left\|\Psi_{i}(k)\right\|_{2}^{2}<1-\left(\frac{\alpha}{\beta}\right)^{2}
$$

Then, the closed-loop system (11) is U.P.A.S., and system (10) is U.P.A.C. with respect to $(\alpha, \beta)$ with $0<\alpha<\beta$. Proof. In fact, by (12), noting that $0<\alpha<\beta$, then, $\forall \varepsilon \in$ $\left(0, \alpha^{2} / \beta^{2}\right)$, there exist scalars $v_{1} \in\left[\alpha^{2} / \beta^{2}, 1\right]$ and $v_{2}>1$ such that

$\sup _{k \in \mathbb{Z}}\left\|\Psi_{0}(k)\right\|_{2}^{2}+v_{2} \sup _{k \in \mathbb{Z}} \sum_{i=1}^{d}\left\|\Psi_{i}(k)\right\|_{2}^{2}<v_{1}-\left(\frac{\alpha}{\beta}\right)^{2}+\varepsilon<v_{1}$.

Thus, there exists a positive definite matrix $P$ such that $\lambda_{\min }(P)=v_{1} \lambda_{\max }(P)$. Choose $V(k, x(k))=x^{T}(k) P x(k)$, $W_{1}(|x(k)|)=\lambda_{\min }(P) x^{T}(k) x(k), \quad$ and $\quad W_{2}(|x(k)|)=$ $\lambda_{\max }(P) x^{T}(k) x(k)$. It is obvious that

$$
W_{1}(|x(k)|) \leq V(k, x(k)) \leq W_{2}(|x(k)|) .
$$

Let $\hat{P}(s)=v_{2} s$ for $s \geq 0$. Then $\hat{P}(s)>s$ for $s \geq 0$. For all $i \in\{1, \ldots, d\}$, if $V(k-i, x(k-i))<\hat{P}(V(k+1, x(k+1)))$, then, $\|x(k-i)\|_{2}^{2}<\|x(k+1)\|_{2}^{2} v_{2} / v_{1}$, and it follows (11) that

$$
\begin{aligned}
\|x(k+1)\|_{2}^{2} \leq & \sup _{k \in \mathbb{Z}}\left\|\Psi_{0}(k)\right\|_{2}^{2}\|x(k)\|_{2}^{2} \\
& +\sup _{k \in \mathbb{Z}} \sum_{i=1}^{d}\left\|\Psi_{i}(k)\right\|_{2}^{2}\|x(k-i)\|_{2}^{2} \\
\leq & \sup _{k \in \mathbb{Z}}\left\|\Psi_{0}(k)\right\|_{2}^{2}\|x(k)\|_{2}^{2} \\
& +\frac{v_{2} \sup _{k \in \mathbb{Z}} \sum_{i=1}^{d}\left\|\Psi_{i}(k)\right\|_{2}^{2}}{v_{1}}\|x(k+1)\|_{2}^{2} .
\end{aligned}
$$

Consequently,

$$
-\|x(k)\|_{2}^{2} \leq \frac{v_{2} \sup _{k \in \mathbb{Z}} \sum_{i=1}^{d}\left\|\Psi_{i}(k)\right\|_{2}^{2}-v_{1}}{v_{1} \sup _{k \in \mathbb{Z}}\left\|\Psi_{0}(k)\right\|_{2}^{2}}\|x(k+1)\|_{2}^{2} .
$$

Let $\delta=\frac{v_{1}-\sup _{k \in \mathbb{Z}}\left\|\Psi_{0}(k)\right\|_{2}^{2}-v_{2} \sup _{k \in \mathbb{Z}} \sum_{i=1}^{d}\left\|\Psi_{i}(k)\right\|_{2}^{2}}{\sup _{k \in \mathbb{Z}}\left\|\Psi_{0}(k)\right\|_{2}^{2}}$. Since scalar $\varepsilon \in$ $\left(0, \alpha^{2} / \beta^{2}\right)$ is arbitrary, thus, $\delta>\frac{\alpha^{2}}{\beta^{2}-\alpha^{2}}>0$, and

$$
\begin{aligned}
\Delta V(k, X) & =x^{T}(k+1) \operatorname{Px}(k+1)-x^{T}(k) P x(k) \\
& \leq-\lambda_{\max }(P) \frac{\alpha^{2}}{\beta^{2}-\alpha^{2}}\|x(k+1)\|_{2}^{2} .
\end{aligned}
$$

Then, conditions (i), (ii) and (iii) $)_{b}$ of Theorem 3.3 are all satisfied, and hence, the conclusion follows.

Remark 5.4: In Theorem 3.1, Corollary 3.2, Theorem 3.3 and Theorem 4.1, there is a relation between $V(k+$ $s, x(k+s))\left(s \in I_{d}^{1}\right)$ and $V(k+1, x(k+1))$, namely, "provided $\mathscr{R}(V(k+s, x(k+s)), V(k+1, x(k+1)))$ ", where $\mathscr{R}(\cdot, \cdot)$ defines a relation. We call this relation as the $\mathscr{R}$-relation. The condition (ii), (ii) ${ }^{\prime}$, (iii) $_{a}$ and (iii) $)_{b}$ describe the constraint on $\Delta V(k, X)$ under the $\mathscr{R}$-relation, but no constrain on $\Delta V(k, X)$ without $\mathscr{R}$-relation. Thus, the condition that the constraint on $\Delta V(k, X)$ holds not only with but also without the $\mathscr{R}$-relation, is more restrictive than the condition that the constraint on $\Delta V(k, X)$ holds only with the $\mathscr{R}$-relation. Therefore, we can obtain a class of particular cases of Theorem 3.3 with conditions (i), (ii), either (iii) $)_{a}$ or (iii) $)_{b}$, which in fact are corresponding to the well-known Lyapunov-like theorems.

\section{ILLUSTRATIVE NUMERICAL EXAMPLE}

To illustrate the effectiveness of the obtained results in previous sections, we consider the following nonlinear discrete system with input time delay:

$$
\begin{aligned}
x(k+1)= & 1.44 x(k)-x^{3}(k)+0.069 u(k) \\
& +0.031 u(k-1), x(k) \in[-1.2,1.2]
\end{aligned}
$$

Assume that $\alpha=0.45$ and $\beta=0.60$. To obtain the zero solution $x(k)=0$ in U.P.A.S with $(\alpha, \beta)$, adopt the following fuzzy control law:

$$
\begin{array}{ll}
R_{1}: & \text { IF } x \text { is about } \pm 1.2, \text { THEN } \\
& u=F_{1} x(k), \\
R_{2}: & \text { IF } x \text { is about } 0, \text { THEN } \\
& u=F_{2} x(k) .
\end{array}
$$

The references on fuzzy control can be found in [11], [12]. Then, the overall control law is

$$
u(k)=\sum_{i=1}^{2} \mu_{i} F_{i} x(k) .
$$

where $\mu_{1}=\frac{x^{2}}{1.44}$ and $\mu_{2}=1-\mu_{1}$ are both membership functions, as shown in Fig. 2. The control gain matrices are 
designed to be $F_{1}=-0.0694$ and $F_{2}=-18.9114$. Then, the closed-loop system can be expressed as follows:

$$
\begin{aligned}
x(k+1)= & \left(1.44-x^{2}(k)+0.069 \sum_{i=1}^{2} \mu_{i} F_{i}\right) x(k) \\
& +0.031 \sum_{i=1}^{2} \mu_{i} F_{i} x(k-1) .
\end{aligned}
$$

Denote discriminant function by

$g(x(k))=\left(1.44-x^{2}(k)+0.069 \sum_{i=1}^{2} \mu_{i} F_{i}\right)^{2}+\left(0.031 \sum_{i=1}^{2} \mu_{i} F_{i}\right)^{2}$.

The profile of $g(x)$ is illustrated in Fig. 3. We can calculate that $g(x) \leq 0.3619<1-\alpha^{2} / \beta^{2}=0.4375$ for $x \in[-1.2,1.2]$. by (12) and Corollary 5.3, system (13) is U.P.A.C. with respect to $(\alpha, \beta)$. The state curve with initial values $x(-1)=$ $0.3, x(0)=0.4$ of system (13) with and without fuzzy controller (14) are shown in Fig. 4. Without fuzzy controller, i.e., $u(k)=0$, the zero solution is unstable, and the nonlinear discrete system converges to $x(k) \approx 0.6633>\beta$; whereas, with fuzzy controller (14), the closed-loop system is U.P.A.S. with $(\alpha, \beta)$.

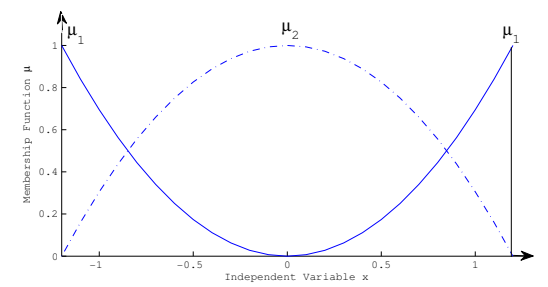

Fig. 2. The membership functions of $\mu_{1}$ and $\mu_{2}$

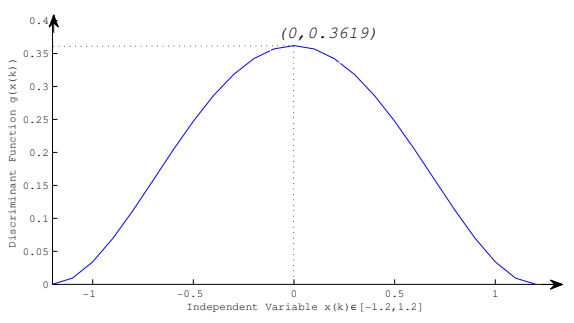

Fig. 3. The profile of $g(x(k))$

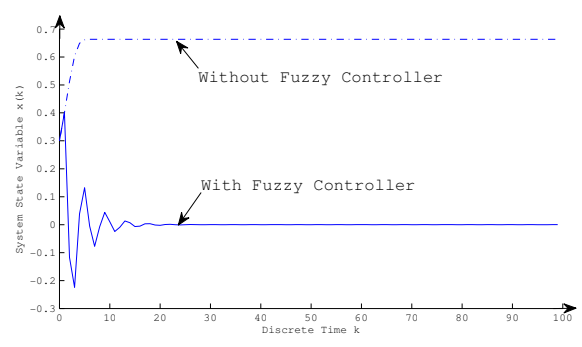

Fig. 4. The state curve of system (13) with and without fuzzy controller (14)

\section{CONCLUSIONS}

Motivated by the idea in [9], we studied the Razumikhintype theorems on practical asymptotic stability for a class of discrete time-delay system. Some easy testing criteria for the uniform practical asymptotical stability are derived via Lyapunov function and Razumikhin technique. Estimations of the solution boundary and arrival time of the solution are also investigated. In addition, the proposed theorems are used to study the practical controllability for a general class of nonlinear discrete systems with input time delay. Finally, a numerical example is present to illustrate the effectiveness of the proposed results. We believe the results in this paper are useful for the study networked control systems.

\section{ACKNOWLEDGMENTS}

The authors gratefully acknowledge the contribution of National Research Organization and reviewers' comments.

\section{REFERENCES}

[1] J. Lasalle and S. Lefshetz, Stability by Lyapunov direct method and application, Academic Press, New York, 1961.

[2] V. Lakshmikantham, S. Leela and A. A. Martynyuk, Practical Stability of Nonlinear Systems, World Scientific, Singapore, 1990.

[3] D. F. Delchamps, "Stabilizing a linear system with quantized state feedback", IEEE Transactions on Automatic Control, vol. 35, no.8, 1990, pp 916-924.

[4] J. H. Chou, S. H. Chen and I. R. Horng, "Robust stability bound on linear time-varying uncertainties for linear digital control systems under finite wordlength effects", JSMS International Journal: Series C, vol. 39, no.4, 1996, pp 767-771.

[5] N. Elia and S. K. Mitter, "Stabilization of linear systsems with limited information", IEEE Transactions on Automatic Control, vol. 46, no.9, 2001, pp 1384-1400.

[6] F. Fagnani and S. Zampieri, "Stability analysis and synthesis for scalar linear systems with a quantized feedback", IEEE Transactions on Automatic Control, vol. 48, no.9, 2003, pp 1569-1584.

[7] C. Hou and J. Qian, "Decay estimates for applictions of Razumikhintype theorems", Automatica, vol, 34, no.7, 1998, pp 921-924.

[8] F. Blanchini and E. P. Ryan, "A Razumikhin-type lemma for functional differential equations with application to adaptive control", Automatica, vol. 35, 1999, pp 809-818.

[9] S. Zhang and M. P. Chen, "A new razumikhin theorem for delay difference equations", Computers and Mathematics with Applications, vol. 36, 1998, pp 405-412.

[10] S. Zhang, "A new technique in stability of infinite delay differential equations", Computers and Mathematics with Applications, vol. 44, 2002, pp 1275-1287.

[11] T. Takagi and M. Sugeno, "Fuzzy identification of system and its applictions to modeling and control, IEEE Transactions on Systems, Man, and Cybernetics, vol. 15, no.1, 1985, pp 116-132.

[12] R. Babuska, Fuzzy Modeling For Control, Kluwer Academic Publishers, 1998. 